\title{
Megalencephalic leukoencephalopathy with subcortical cysts
}

\author{
Characterization of disease variants
}

Eline M.C. Hamilton, MD, Pinar Tekturk, MD, Fia Cialdella, BSc, Diane F. van Rappard, MD, Nicole I. Wolf, MD, PhD, Cengiz Yalcinkaya, MD, Ümran Çetinçelik, MD, Ahmad Rajaee, MD, Ariana Kariminejad, MD, Justyna Paprocka, MD, PhD, Zuhal Yapici, MD, Vlatka Mejaški Bošnjak, MD, PhD, and Marjo S. van der Knaap, MD, PhD On behalf of the MLC Research Group

Neurology ${ }^{\circledR}$ 2018;90:e1395-e1403. doi:10.1212/WNL.0000000000005334

\begin{abstract}
Objective

To provide an overview of clinical and MRI characteristics of the different variants of the leukodystrophy megalencephalic leukoencephalopathy with subcortical cysts (MLC) and identify possible differentiating features.
\end{abstract}

\section{Methods}

We performed an international multi-institutional, cross-sectional observational study of the clinical and MRI characteristics in patients with genetically confirmed MLC. Clinical information was obtained by questionnaires for physicians and retrospective chart review.

\section{Results}

We included 204 patients with classic MLC, 187 of whom had recessive mutations in MLC1 (MLC1 variant) and 17 in GLIALCAM (MLC2A variant) and 38 patients with remitting MLC caused by dominant GLIALCAM mutations (MLC2B variant). We observed a relatively wide variability in neurologic disability among patients with classic MLC. No clinical differences could be identified between patients with MLC1 and MLC2A. Patients with MLC2B invariably had a milder phenotype with preservation of motor function, while intellectual disability and autism were relatively frequent. Systematic MRI review revealed no MRI features that distinguish between MLC1 and MLC2A. Radiologic improvement was observed in all patients with MLC2B and also in 2 patients with MLC1. In MRIs obtained in the early disease stage, absence of signal abnormalities of the posterior limb of the internal capsule and cerebellar white matter and presence of only rarefied subcortical white matter instead of true subcortical cysts were suggestive of MLC2B.

\section{Conclusion}

Clinical and MRI features did not distinguish between classic MLC with $M L C 1$ or GLIALCAM mutations. Absence of signal abnormalities of the internal capsule and cerebellar white matter are MRI findings that point to the remitting phenotype.

\author{
Correspondence \\ Dr. van der Knaap \\ ms.vanderknaap@vumc. \\ $\mathrm{nl}$
}

- CME Course

NPub.org/cmelist

From the Department of Child Neurology and Amsterdam Neuroscience (E.M.C.H., F.C., D.F.v.R., N.I.W., M.S.v.d.K.), VU University Medical Center, Amsterdam, the Netherlands; Division of Child Neurology, Department of Neurology, Istanbul Faculty of Medicine (P.T., Z.Y.), and Division of Child Neurology, Department of Neurology, Cerrahpasa Medical School (C.Y.), Istanbul University; clinical geneticist in private practice (Ü.C..), Merkez Mahallesi, Istanbul, Turkey; Kariminejad-Najmabadi Pathology \& Genetics Center (A.R., A.K.), Tehran, Iran; Department of Pediatric Neurology (J.P.), School of Medicine in Katowice, Medical University of Silesia, Katowice, Poland; Department of Neuropediatrics (V.M.B.), Children's Hospital Zagreb, School of Medicine, University of Zagreb, Croatia; and Department of Functional Genomics (M.S.v.d.K.), Center for Neurogenomics and Cognitive Research, Amsterdam Neuroscience, VU University, Amsterdam, the Netherlands.

Coinvestigators are listed at links.Iww.com/WNL/A357.

Go to Neurology.org/N for full disclosures. Funding information and disclosures deemed relevant by the authors, if any, are provided at the end of the article.

The Article Processing Charge was funded by VU University Medical Center.

This is an open access article distributed under the terms of the Creative Commons Attribution-NonCommercial-NoDerivatives License 4.0 (CC BY-NC-ND), which permits downloading and sharing the work provided it is properly cited. The work cannot be changed in any way or used commercially without permission from the journal. 


\section{Glossary}

CFCS = Communication Function Classification System; GMFCS = Gross Motor Function Classification System; MACS = Manual Ability Classification System; MLC = megalencephalic leukoencephalopathy with subcortical cysts; MLC1 = classic megalencephalic leukoencephalopathy with subcortical cysts caused by recessive MLC1 mutations; MLC2A = classic megalencephalic leukoencephalopathy with subcortical cysts caused by recessive GLIALCAM mutations; MLC2B = remitting megalencephalic leukoencephalopathy with subcortical cysts caused by dominant GLIALCAM mutations; SUDEP = suspected sudden unexpected death in epilepsy.

Megalencephalic leukoencephalopathy with subcortical cysts (MLC) is an infantile-onset inherited disorder characterized by cerebral white matter edema. ${ }^{1-3}$ MRI shows diffuse signal abnormalities of the cerebral hemispheric white matter. The swelling of the abnormal cerebral white matter is most prominent in the first few years of life. ${ }^{1}$ Subcortical cysts are typically located in the anterior temporal region and less consistently elsewhere. ${ }^{4}$ Two different MLC phenotypes can be distinguished: a classic, deteriorating phenotype, and a remitting phenotype. ${ }^{5}$ Classic MLC is caused by recessive mutations in the $M L C 1$ gene (MIM 605908) in the majority of cases: this variant is called MLC1 (MIM 604004). ${ }^{6}$ Classic MLC caused by recessive GLIALCAM mutations (also known as HEPACAM, MIM $613925)$ is called MLC2A. ${ }^{6}$ Patients with remitting MLC or MLC2B (MIM 613926) have dominant GLIALCAM mutations. ${ }^{7}$ Classic MLC starts with increasing macrocephaly in the first year of life; after a few years, patients develop neurologic signs, most commonly ataxia, spasticity, and epilepsy. ${ }^{1}$ Mortality is thought to be low, although no systematic study on survival has been performed. Remitting MLC initially resembles classic MLC: patients generally present with progressive macrocephaly and may have developmental delay, but neurologic deterioration does not occur and the MRI abnormalities improve or normalize.

Among patients with MLC1, a relatively broad variation in disease severity has been described, also for siblings and unrelated patients with the same mutations. ${ }^{8,9}$ So far, no clinical differences have been recognized between patients with MLC2A vs MLC1. It is unknown whether MRI features allow distinction among MLC1, MLC2A, and MLC2B before improvement occurs in the latter. MLC is a rare disorder and large studies on the disease course are scarce. ${ }^{8,10,11}$ Systematic review of MRI characteristics has not been performed. The objective of the current study is to identify potential different and perhaps discriminating clinical and MRI features for different MLC variants, aiming at improved clinical recognition and avoidance of unnecessary genetic testing.

\section{Methods}

\section{Study design}

We performed an international, cross-sectional observational multicenter study among all patients with genetically proven MLC enrolled in the Amsterdam database of leukoencephalopathies between January 1991 and January 2017. The database contains patients from over the world referred to VU University Medical Center for MRI review and mutational analysis. A prerequisite for genetic testing was review of clinical and MRI data in Amsterdam. ${ }^{1}$ We performed analysis of the $M L C 1$ gene as previously described, ${ }^{12}$ if necessary including analysis of $M L C 1$ copy $\mathrm{DNA}^{12}$ and multiplex ligation-dependent probe amplification. ${ }^{13}$ Analysis of the GLIALCAM gene was also performed as previously described. ${ }^{7}$ By DNA analysis in the parents, we confirmed that recessive variants were biallelic.

\section{Standard protocol approvals, registrations, and patient consents}

We received approval from the ethical standards committee for clinical evaluation, systematic MRI review, and review of the DNA findings and obtained informed consent from the patients/guardians.

\section{Clinical information}

We analyzed clinical information available at the start of the study, supplemented by information obtained via a clinical questionnaire for physicians. We focused on measures that could be retrospectively assessed, including head circumference, motor deterioration (especially loss of ambulation), seizures, and death. As formal, standardized assessment of motor and cognitive development, autistic features, behavioral problems, and current cognitive function was not feasible, these items were subjectively assessed by physicians and caretakers or derived from medical records. The patients' status at last examination was assessed by quantitative, validated, and widely used 5-level classification systems for gross motor function (Gross Motor Function Classification System [GMFCS]), manual ability (Manual Ability Classification System [MACS]), and communication function (Communication Function Classification System $[\mathrm{CFCS}]) .{ }^{14}$ Scores on these scales range from I (no limitations) to $\mathrm{V}$ (severe limitations; table e-1, links.lww.com/WNL/A356). Patients with comorbidities affecting neurologic function were excluded from the study.

\section{MRI scoring}

We scored all available MRIs according to a standardized protocol. ${ }^{15}$ Specifically, cysts were defined as areas with the same signal intensity of CSF on all pulse sequences including fluid-attenuated inversion recovery, while in rarefaction the signal intensity was close but not the same. ${ }^{16}$ 


\section{Statistical analysis}

We used summary statistics to describe the clinical characteristics. Results were reported by means \pm SD for continuous variables that were normally distributed, and median with 25 th and 75th percentiles for non-normally distributed data and ranges. We performed time-to-event analysis of the events "start of motor deterioration," "start of cognitive decline," "first seizure," "loss of walking without support," "loss of walking with or without support," and "death," with age as time variable. Individuals in whom the respective event had not occurred at the last follow-up were indicated as censored for the respective analysis. Nonambulatory patients younger than 18 months were not included in the analysis of loss of ambulation. We estimated the median ages at which the events had occurred by plotting Kaplan-Meier curves. Group differences regarding disease variant were analyzed with the log-rank test. We performed linear regression analysis to compare GMFCS, MACS, and CFCS scores in relation to age per disease variant. Statistical analysis was performed using SPSS version 22 (IBM, Armonk, NY) and GraphPad Prism version 6.07 (San Diego, CA).

\section{Results}

\section{Patients}

A total of 245 patients with MLC from 207 families were diagnosed with MLC by DNA analysis. Three patients were excluded because of comorbidity (Turner syndrome, asphyxia, pituitary adenoma). In the case of limited clinical information due to loss to follow-up or nonresponse, patients were selectively included in the analyses on the basis of availability of information. Throughout the Results section we report the number of patients included in the different analyses in parentheses or in the respective tables. In total, 242 patients with MLC, of whom 187 had MLC1, 17 had MLC2A, and 38 had MLC2B, were included.

\section{Clinical characteristics}

Details are presented in table 1. Macrocephaly in the first year of life was the most common first disease sign and had been present in almost all patients in infancy. Macrocephaly persisted in more than half of patients. Secondary normocephaly was particularly common in patients with MLC2B. Initial motor development was reported as mildly delayed in the majority of patients and normal in a smaller number. All patients except 12 patients with MLC1 achieved unsupported walking, at a mean age of 16 months ( \pm 8 , range 10-72). Initial cognitive development was reported as normal in just over half of patients with MLC1, MLC2A, and MLC2B. Kaplan-Meier curves on start of motor deterioration indicated that both patients with MLC1 and patients with MLC2A showed the first signs of motor deterioration at a median age of 5 years (range 6 months- 41 years); there were no significant differences between the curves $(p=$ 0.98; figure 1A). None of the patients with MLC2B had a decline of motor function; in 34\% (10/29), some clumsiness remained.

Loss of walking without support occurred in the majority of patients with MLC1 and MLC2A at ages ranging from 18 months to 43 years; Kaplan-Meier curves showed no significant differences between MLC1 and MLC2A ( $p=0.66$; figure 1B). Median age at loss of walking without support was estimated to be 15 years in MLC1 and 11 years in MLC2A. Patients with MLC2B all remained ambulatory.

Loss of walking with or without support (full wheelchair dependency) occurred in fewer than half of patients with MLC1 and MLC2A and none of the patients with MLC2B. There were no significant differences between patients with MLC1 and patients with MLC2A $(p=0.58$; figure $1 \mathrm{C})$. Patients were most likely to become wheelchair dependent before the age of 15 years; after this age, patients generally remained ambulatory, with or without support.

Behavioral problems were relatively common for all MLC variants; autism was most common among patients with MLC2B. Delayed onset cognitive decline was reported in almost half of patients with MLC1 and MLC2A, and none of the patients with MLC2B. However, stable cognitive impairment was reported in a quarter of patients with MLC2B. The age at start of cognitive decline did not statistically differ between MLC1 and MLC2A $(\log \operatorname{rank} p=0.10)$. The patients' cognitive levels at time of the inventory are presented in table 1 .

At the time of clinical phenotyping, 109 patients had had at least one seizure, of whom the majority had occasional seizures that were generally well-controlled with medication (table 1). Kaplan-Meier curve analysis indicated that approximately $75 \%$ of patients with MLC1 and MLC2A had had one or multiple seizures by age 20 years; the median age at the first seizure was 3 years, mode 2 years (figure 1D). Seizures were less common among patients with MLC2B $(n=4 / 34)$. There were no significant differences in onset of seizures between patients with MLC1 and MLC2A $(p=0.64)$.

Overview of GMFCS, MACS, and CFCS scores in relation to age and disease variant showed that there was a wide variability in clinical severity among patients with MLC1 and MLC2A (figure 2). Linear regression analysis showed no significant differences between the patients with MLC1 and MLC2A regarding GMFCS $(p=0.30)$, MACS $(p=0.18)$, and CFCS scores $(p=0.89)$. Gross motor function and dexterity were generally well-preserved in patients with MLC2B and communication function to a lesser degree.

Several patients with classic MLC had very mild disease: $25 \%$ $(17 / 68)$ of patients with MLC1 and 45\% (5/11) with MLC2A who were at least 12 years at latest clinical evaluation were able to walk without support and had normal cognition or only learning problems. For ages 12 years and above, 32\% $(19 / 59)$ of patients with MLC1 and 50\% (5/10) with MLC2A had GMFCS, MACS, and CFSC scores of I or II.

Survival was assessed in all 242 patients; 7 were deceased (figure 1E). Five deceased patients had MLC1: 4 male patients died at age 7-22 years and 1 female patient at age 56 years. 
Table 1 Clinical characteristics at latest phenotyping

\begin{tabular}{|c|c|c|c|}
\hline & MLC1 & MLC2A & MLC2B \\
\hline \multicolumn{4}{|l|}{ Study characteristics } \\
\hline No. of patients & 187 & 17 & 38 \\
\hline Male/female & $102 / 85$ & $9 / 8$ & $27 / 11$ \\
\hline Median age at latest phenotyping, y (quartiles) & $13(6-19)$ & $15(7-23)$ & $7(2-13)$ \\
\hline \multicolumn{4}{|l|}{ Head circumference, \% (n/N) } \\
\hline$\geq 2$ SD below age 2 years & $99(121 / 122)$ & $88(14 / 16)$ & $88(30 / 34)$ \\
\hline Currently $\geq 2$ SD & $93(122 / 131)$ & $73(11 / 15)$ & $56(15 / 27)$ \\
\hline \multicolumn{4}{|l|}{ Walking without support, \% (n/N) } \\
\hline Achieved $\leq 18 \mathrm{mo}$ & $63(88 / 138)$ & $65(11 / 17)$ & $86(30 / 35)$ \\
\hline Achieved >18 mo & $28(38 / 138)$ & $35(6 / 17)$ & $14(5 / 35)$ \\
\hline Not achieved & $9(12 / 138)$ & $0(0 / 17)$ & $0(0 / 35)$ \\
\hline \multicolumn{4}{|l|}{ Seizures } \\
\hline No history of seizures & $38(50 / 132)$ & $31(5 / 16)$ & $88(30 / 34)$ \\
\hline Single seizure & $7(9 / 132)$ & $19(3 / 16)$ & $3(1 / 34)$ \\
\hline Well-controlled epilepsy & $46(61 / 132)$ & $44(7 / 16)$ & $6(2 / 34)$ \\
\hline Poorly controlled epilepsy & $9(12 / 132)$ & $6(1 / 16)$ & $3(1 / 34)$ \\
\hline \multicolumn{4}{|l|}{ History of behavioral problems, \% (n/N) } \\
\hline Autistic features & $9(8 / 86)$ & $0(0 / 15)$ & $25(9 / 36)$ \\
\hline Other behavioral problems & $29(31 / 107)$ & $20(3 / 15)$ & $28(9 / 32)$ \\
\hline Psychiatric diagnosis & $14(15 / 111)$ & $7(1 / 14)$ & $3(1 / 30)$ \\
\hline \multicolumn{4}{|l|}{ Current cognitive function, $\%(\mathrm{n} / \mathrm{N})$} \\
\hline Normal & $37(48 / 129)$ & $31(5 / 16)$ & $73(24 / 33)$ \\
\hline Learning difficulties & $21(27 / 129)$ & $25(4 / 16)$ & $15(5 / 33)$ \\
\hline Mild mental retardation & $26(34 / 129)$ & $31(5 / 16)$ & $12(4 / 33)$ \\
\hline Severe mental retardation & $16(20 / 129)$ & $13(2 / 16)$ & $0(0 / 33)$ \\
\hline
\end{tabular}

Abbreviations: MLC1 = classic megalencephalic leukoencephalopathy with subcortical cysts caused by recessive MLC1 mutations; MLC2A = classic megalencephalic leukoencephalopathy with subcortical cysts caused by recessive GLIALCAM mutations; MLC2B = remitting megalencephalic leukoencephalopathy with subcortical cysts (caused by dominant GLIALCAM mutations).

Causes of death were respiratory insufficiency after neurologic deterioration following head trauma, suspected sudden unexpected death in epilepsy (SUDEP), sepsis as complication of surgical procedure, cachexia, and status epilepticus. One deceased male patient with MLC2A and 1 deceased male patient with MLC2B were suspected of SUDEP at ages 23 and 3 years, respectively.

To assess a possible genotype-phenotype correlation in patients with MLC1 and MLC2A, we compared loss of ambulation, wheelchair dependency, and seizures in groups of patients with 3 or more patients from 2 or more families with the same mutations, who were at least 10 years of age at the clinical inventory. The number of patients with MLC2A was too small for evaluation. Six groups of informative patients with MLC1 were available for comparison; characteristics were very divergent within each group (table e-2, links.lww.com/WNL/ A356), arguing against a genotype-phenotype correlation.

\section{MRI characteristics}

MRIs were available for 187 patients and for 53 patients 1 or more follow-up scans were available; we evaluated a total of 268 MRIs. Patients had their first MRI at a median age of 2 years (range 4 months-55 years). Follow-up scans were obtained at a median age of 5 years (range 11 months-43 years). The median interval between the first and last MRIs was 4 years (range 3 months -15 years). 

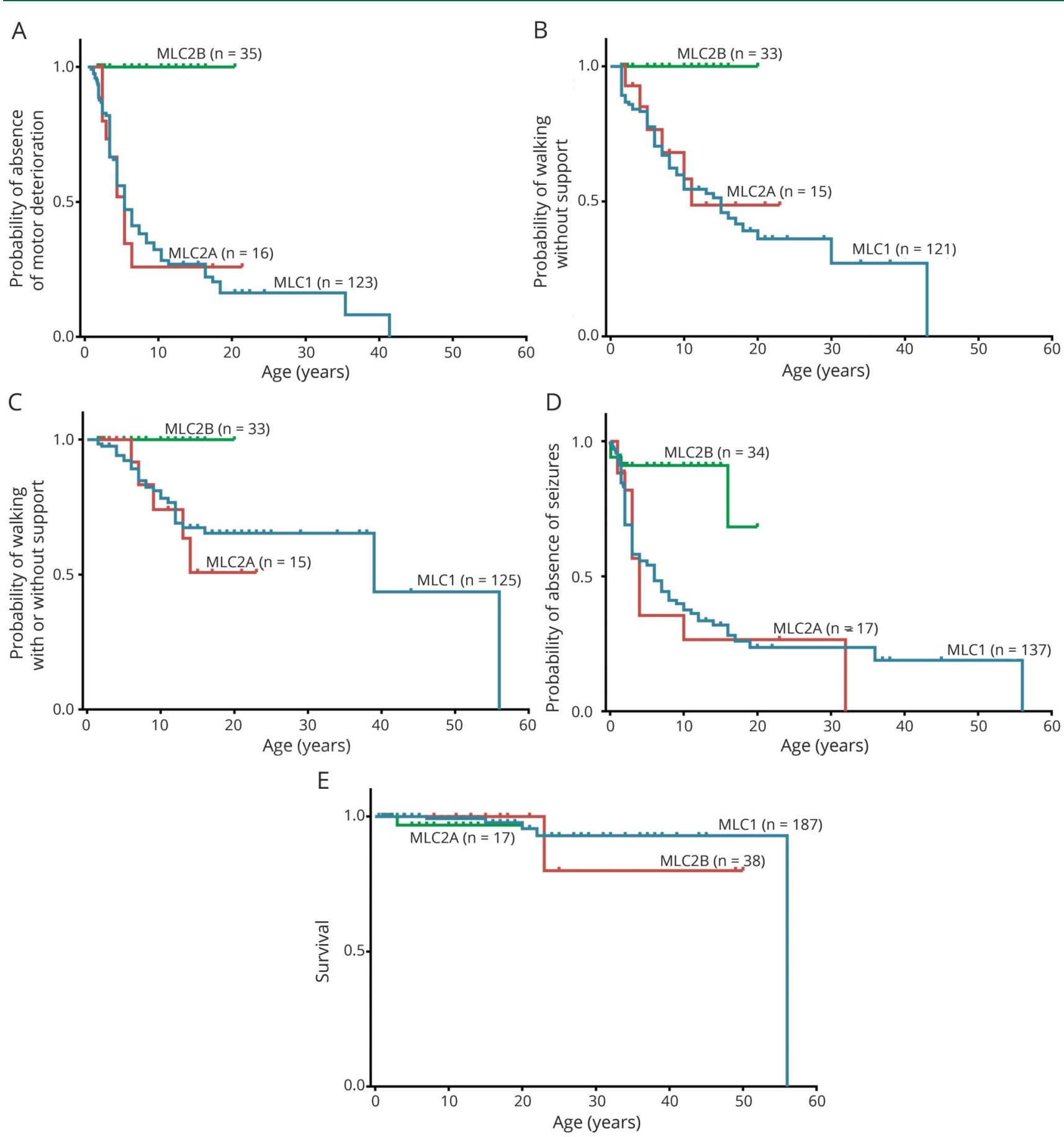

Kaplan-Meier plots on (A) onset of motor deterioration, (B) loss of walking without support, (C) loss of walking with or without support (full wheelchair dependency), (D) onset of seizures, and (E) survival, grouped by disease variant. Censored patients (absence of motor deterioration, still walking without support, still walking with or without support, absence of seizures, or still alive at last follow-up) are indicated by crosses. MLC1 = classic megalencephalic leukoencephalopathy with subcortical cysts caused by recessive MLC1 mutations; MLC2A = classic megalencephalic leukoencephalopathy with subcortical cysts caused by recessive GLIALCAM mutations; MLC2B = remitting megalencephalic leukoencephalopathy with subcortical cysts caused by dominant GLIALCAM mutations.

Details of the first MRIs are presented in table e-3 (links.lww. com/WNL/A356). Extensive, confluent signal abnormalities of the cerebral white matter were invariably present and swelling was observed in all patients, except for one, in whom the first MRI was obtained at 30 years. All patients had sparing of the optic radiation and most patients had sparing in one or more regions of subcortical white matter (figure 3). Several patients also had some regional white matter sparing, mostly in the occipital region (figure 3). There were no clear differences in abnormalities between patients with MLC1 and 
Figure 2 Function levels

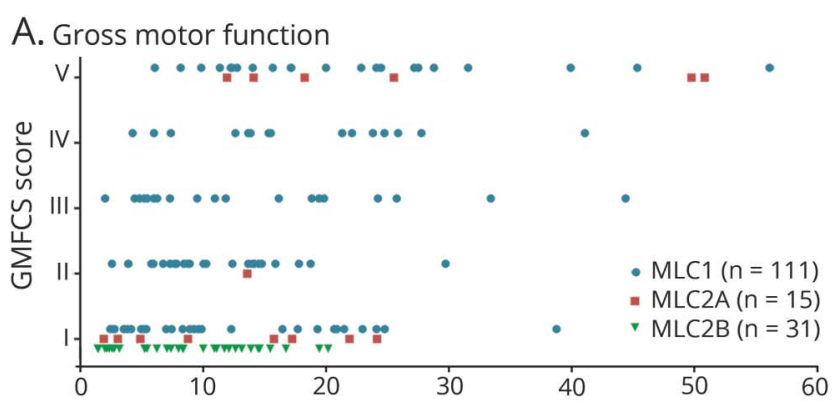

B. Manual ability

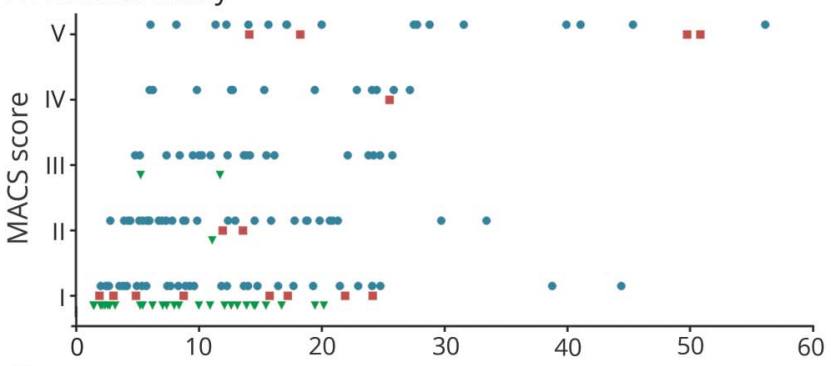

C. Communication function

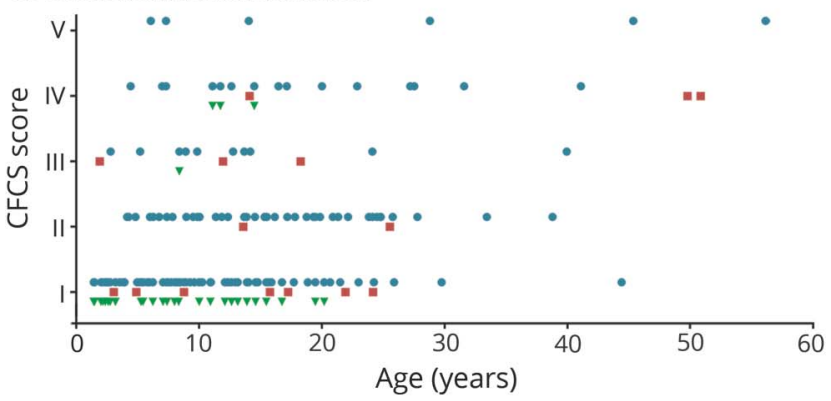

Overview of megalencephalic leukoencephalopathy with subcortical cysts (MLC) patients' scores on (A) the Gross Motor Function Classification System (GMFCS), (B) Manual Ability Classification System (MACS), and (C) Communication Function Classification System (CFCS) in relation to age, grouped by disease variant: MLC1 (classic MLC caused by recessive MLC1 mutations; blue circles), MLC2A (classic MLC caused by recessive GLIALCAM mutations: red squares) and MLC2B (remitting MLC caused by dominant GLIALCAM mutations; green triangles). Scores range from I (no limitations) to V (severe limitations; table e-1, links.Iww.com/WNL/A356).

MLC2A. They all had an abnormal signal of the posterior limb of the internal capsule, while in 54\% of patients with MLC2B, in whom the MRI was obtained before the age of 2 years, the signal of the internal capsule was already normal (figure 3). Widening of the ventricles and enlargement of the subarachnoid space was rare in patients with MLC1 and patients with MLC2A in the early disease stage and more common in MRIs performed in adolescence or adulthood. In patients with MLC2B, some enlargement of the subarachnoid spaces was already observed before the age of 2 years in nearly half of patients.

Subcortical cysts were present in all patients with MLC1 and almost all patients with MLC2A. Forty-three percent of patients with MLC2B did not have true cysts, but only near-cystic rarefaction of subcortical white matter. Cysts or near-cystic rarefaction were almost invariably present in both anterior temporal lobes. Additional cysts were located in the frontal and parietal lobes, but never in the occipital lobe. Near-cystic rarefaction of subcortical white matter could be located in all 4 lobes.

The majority of patients with MLC1 and patients with MLC2A ( $85 \%)$ had mild signal abnormalities of the cerebellar white matter, which were less pronounced than the cerebral white matter signal abnormalities (figure 3). Patients with MLC2B never had cerebellar white matter signal abnormalities; a few patients younger than 10 months showed some delay in myelination featured by a hyperintense rim of the subcortical cerebellar white matter (figure e-1, links.lww. com/WNL/A355). The cerebellar white matter was never swollen. Almost all patients with MLC1, MLC2A, and MLC2B had mild signal abnormalities in the brainstem.

\section{Change over time}

Details are presented in table 2. Normalization of the signal and reversal of the swelling of the white matter only occurred in patients with MLC2B. In patients with MLC1 and patients with MLC2A, the white matter signal abnormalities persisted or increased, with the exception of 2 patients with MLC1 who showed improvement. The number of cysts generally remained the same or increased among patients with MLC1 and MLC2A; in MLC2B, a decrease of cysts and overall quantity of cystic white matter generally occurred, although in all but one patient some cysts or near-cysts persisted.

Existing signal abnormalities of the posterior limb of the internal capsule and cerebellar white matter never improved in patients with MLC1 and MLC2A. The few patients with MLC2B who had a rim of slightly abnormal cerebellar white matter on the initial MRI all showed normalization over time.

\section{Discussion}

MLC is caused by mutations in MLC1 or GLIALCAM. MLC1 is an astrocyte-specific membrane protein involved in brain ion and water homeostasis. ${ }^{3,17}$ GlialCAM is a chaperone of MLC1 ensuring its localization in astrocytic endfeet. ${ }^{7,18,19}$ So, both MLC1 and GLIALCAM mutations affect MLC1 protein function. In addition, GlialCAM is involved in transport of other proteins, such as connexin 43 and the chloride channel $\mathrm{ClC} 2{ }^{20,21}$ Recessive CLCN2 mutations cause a leukoencephalopathy with childhood or adult onset, characterized by mild motor dysfunction and often retinopathy. ${ }^{22}$ Brain MRI shows restricted diffusion in the posterior limbs of internal capsules, brainstem structures, and cerebellar white matter, suggestive of myelin microvacuolization. ${ }^{22}$ Pediatric patients have diffuse, mild cerebral white matter signal abnormalities, while these are limited in adult-onset cases. By contrast, in MLC the cerebral white matter abnormalities are profound and diffusion is highly increased, consistent with myelin macrovacuolization and increased extracellular spaces. ${ }^{23}$ Considering that GlialCAM is supposed to be a chaperone for both MLC1 and $\mathrm{ClC} 2$, one might expect GLIALCAM mutations to cause disease features of both MLC1- and CLCN2-associated 


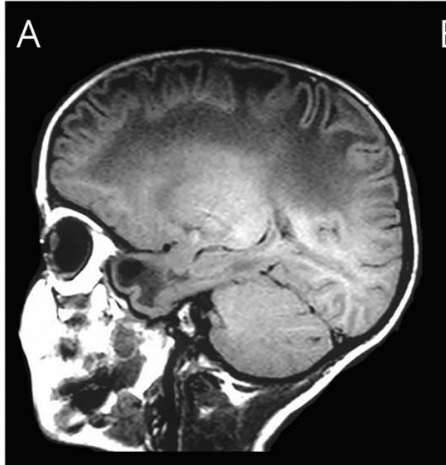

D
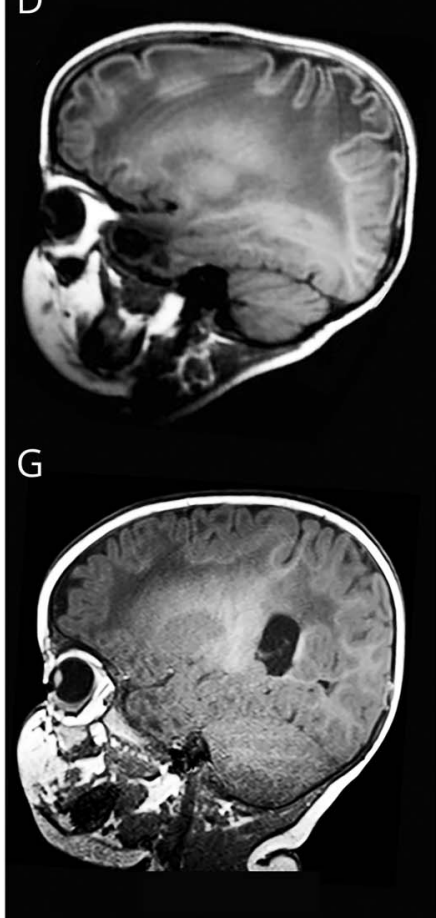

E

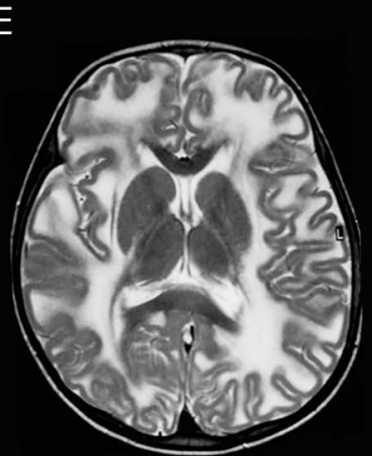

$\mathrm{H}$

\section{B}
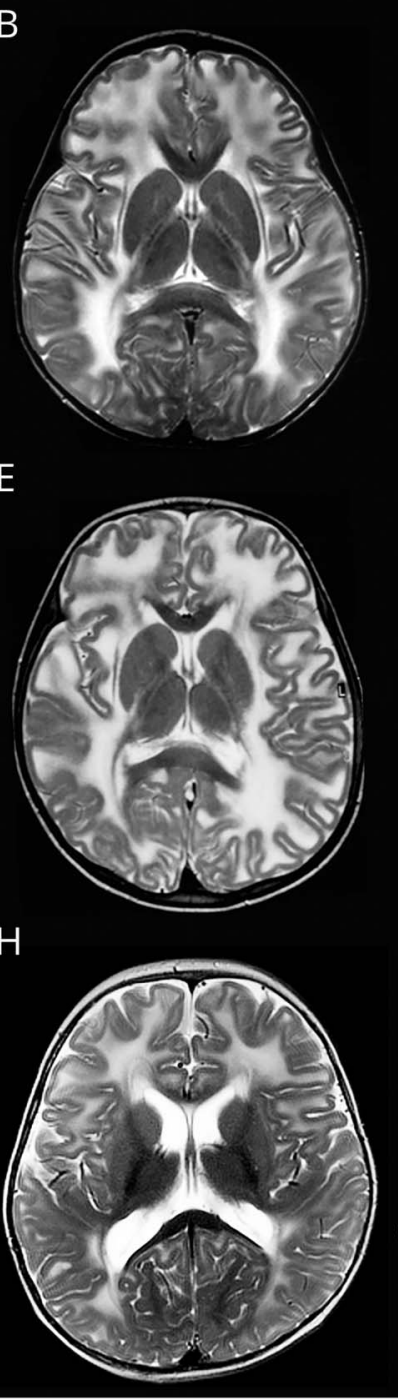

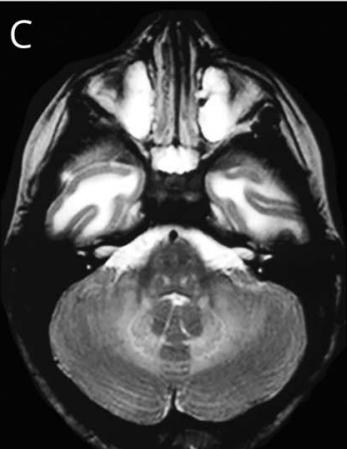

F

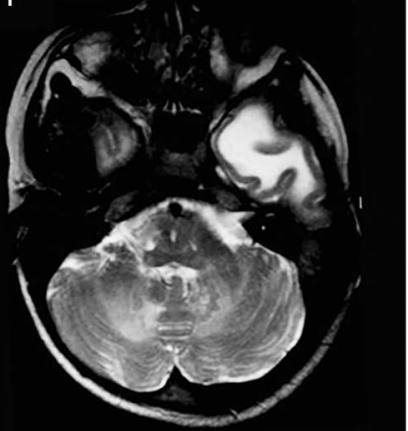

I

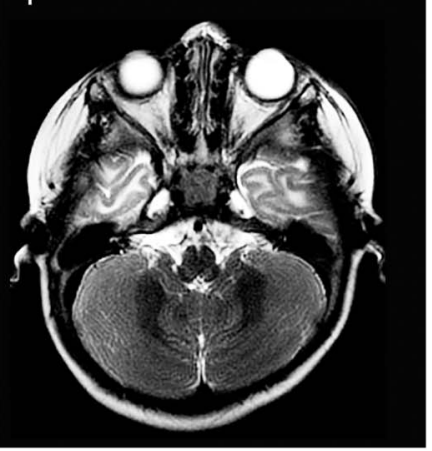

MRI findings in a patient with megalencephalic leukoencephalopathy with subcortical cysts (MLC) caused by recessive $M L C 1$ mutations (MLC1) at age 3 years (top), a patient with MLC caused by recessive GLIALCAM mutations (MLC2A) at age 6 years (middle), and a patient with MLC caused by dominant GLIALCAM mutations (MLC2B) at age 10 months (bottom). Sagittal T1-weighted images show anterior-temporal and frontoparietal cysts in the patient with MLC1 (A), an anterior-temporal cyst in the patient with MLC2A (D), and some near-cystic rarefaction in the anterior-temporal region in the patient with MLC2B (G). The T2weighted axial images of the hemispheres show diffuse white matter abnormalities with some swelling ( $B$, $E, H)$. The sagittal images show some sparing of the subcortical white matter in all patients, especially in the occipital region $(A, D, G)$; in the patient with $\mathrm{MLC} 2 \mathrm{~B}$, the white matter is relatively preserved in the occipital region $(G)$. The patients with classic MLC have a double-line-shaped abnormal signal of the posterior limb of the internal capsule $(B, E)$, while the signal is normal in the patient with MLC2B $(\mathrm{H})$. The T2-weighted axial images of the cerebellum show a mildly hyperintense signal of the cerebellar white matter in the patients with classic MLC (C, F) and a normal signal in the patient with MLC2B (I). diseases. The present study, however, confirms that MLC2A is indistinguishable from MLC1 and by no means shares the MRI features of the CLCN2-related disease. The exact roles of MLC1, GlialCAM, and ClC2 in brain ion and water homeostasis and their interaction remain to be elucidated.

The knowledge obtained from our study concerning a relatively large cohort of patients can help physicians in clinical counseling of patients and families. MLC is a fairly mild and slow disorder, with low mortality compared to other leukodystrophies. ${ }^{24}$ Nevertheless, there is a rather broad variation in clinical severity. Some patients become wheelchair-dependent a few years after onset, while others remain ambulatory during adulthood. An interesting point is that slow motor deterioration often occurs from a few years after presentation onwards, but that patients who are ambulatory with or without support at the age of 15 years most likely remain ambulatory. The study confirms that epilepsy is a common feature in
MLC. ${ }^{25}$ It is puzzling that most patients have well-controlled epilepsy while few patients have refractory epilepsy and $\sim 25 \%$ remain seizure-free. A salient observation is that a considerable proportion of patients with classic MLC exhibit only very mild signs during the disease course; in 2 patients with MLC1, a considerable improvement of the MRI abnormalities occurred. The observed variability in disease severity-also among patients who share the same mutations - may depend on individual differences in compensatory volume regulatory mechanisms. The fact that remarkable improvement of cerebral swelling may occur, as mostly observed in patients with MLC2B but also in a few patients with classic MLC, suggests there is a window of opportunity for therapy.

The multi-institutional and observational nature of this study comes with certain limitations. In the absence of formal testing, measures like cognitive function and presence of autistic features were scored based on subjective assessment by 
Table 2 MRI characteristics: Change over time

\begin{tabular}{|c|c|c|c|}
\hline & MLC1 & MLC2A & MLC2B \\
\hline No. of patients & 29 & 5 & 19 \\
\hline Median interval (range) & 4 y $(3$ mo-13 y) & 13 y $(1-15 y)$ & 3 y $(4$ mo-12 y) \\
\hline \multicolumn{4}{|c|}{ Swelling cerebral WM, \% (n/N) } \\
\hline Normalization & $0(0 / 29)$ & $0(0 / 5)$ & $11(2 / 19)$ \\
\hline Improvement & $21(6 / 29)$ & $40(2 / 5)$ & $78(15 / 19)$ \\
\hline Unchanged & $79(23 / 29)$ & $40(2 / 5)$ & $11(2 / 19)$ \\
\hline Increased & $0(0 / 29)$ & $20(1 / 5)$ & $0(0 / 19)$ \\
\hline \multicolumn{4}{|c|}{ Signal abnormalities cerebral WM, \% (n/N) } \\
\hline Normalization & $0(0 / 29)$ & $0(0 / 5)$ & $21(4 / 19)$ \\
\hline Improvement & $6(2 / 29)$ & $0(0 / 5)$ & $74(14 / 19)$ \\
\hline Unchanged & $73(21 / 29)$ & $40(2 / 5)$ & $5(1 / 19)$ \\
\hline Increased & $21(6 / 29)$ & $60(3 / 5)$ & $0(0 / 19)$ \\
\hline \multicolumn{4}{|c|}{ Signal abnormalities PLIC, \% (n/N) } \\
\hline Not present & $0(0 / 26)$ & $0(0 / 5)$ & $47(9 / 19)$ \\
\hline Normalization & $0(0 / 26)$ & $0(0 / 5)$ & $26(5 / 19)$ \\
\hline Improvement & $8(2 / 26)$ & $0(0 / 5)$ & $11(2 / 19)$ \\
\hline Unchanged & $92(24 / 26)$ & $100(5 / 5)$ & $16(3 / 19)$ \\
\hline \multicolumn{4}{|c|}{ Enlargement ventricles or subarachnoid spaces, \% (n/N) } \\
\hline Not present & $72(21 / 29)$ & $40(2 / 5)$ & $53(10 / 19)$ \\
\hline Unchanged & $4(1 / 29)$ & $0(0 / 5)$ & $0(0 / 19)$ \\
\hline Increased & $24(7 / 29)$ & $60(3 / 5)$ & $47(9 / 19)$ \\
\hline \multicolumn{4}{|l|}{ No. of cysts, \% (n/N) } \\
\hline Not present & $0(0 / 27)$ & $0(0 / 5)$ & $37(7 / 19)$ \\
\hline Decreased & $4(1 / 27)$ & $0(0 / 5)$ & $42(8 / 19)$ \\
\hline Unchanged & $67(18 / 27)$ & $40(2 / 5)$ & $21(4 / 19)$ \\
\hline Increased & $29(8 / 27)$ & $60(3 / 5)$ & $0(0 / 19)$ \\
\hline \multicolumn{4}{|c|}{ Signal abnormalities cerebellar WM, \% (n/N) } \\
\hline Not present & $11(3 / 28)$ & $40(2 / 5)$ & $89(17 / 19)$ \\
\hline Normalization & $3(1 / 28)$ & $0(0 / 5)$ & $11(2 / 19)$ \\
\hline Decreased & $0(0 / 28)$ & $0(0 / 5)$ & $0(0 / 19)$ \\
\hline Unchanged & $86(24 / 28)$ & $60(3 / 5)$ & $0(0 / 19)$ \\
\hline
\end{tabular}

Abbreviations: MLC1 = classic megalencephalic leukoencephalopathy with subcortical cysts caused by recessive MLC1 mutations; MLC2A = classic megalencephalic leukoencephalopathy with subcortical cysts caused by recessive GLIALCAM mutations; MLC2B = remitting megalencephalic leukoencephalopathy with subcortical cysts caused by dominant GLIALCAM mutations; PLIC = posterior limb of the internal capsule; WM = white matter.

physicians and caretakers. The application of time-to-event analysis and standardized scales such as GMFCS resulted in a robust representation of disease progression, but missing data and interobserver differences may have hampered the evaluation of the clinical course. There are, however, no indications that nonresponse was in any way systematic or that censoring in the time-to-event analyses was informative. The study limitations are at least in part compensated by the study size considering the disease is very rare. The available results give an already informative delineation of the clinical spectrum of MLC. Our ongoing data collection will help include larger numbers of patients with MLC2A and extend the follow-up. 
One of the aims of this study was to identify features that can help distinguish different MLC variants in early disease stages. Absence of MRI signal abnormalities of the posterior limb of the internal capsule and cerebellar white matter and presence of only rarefied subcortical white matter instead of actual cysts are features suggestive of MLC2B. This knowledge can be applied to direct genetic testing in patients suspected of the remitting phenotype. No distinguishing features have been identified to discern MLC1 and MLC2A. Considering the much higher prevalence of $M L C 1$ gene defects, in cases of a classic presentation of MLC, this gene should be tested first.

\section{Author contributions}

Eline M.C. Hamilton collected and analyzed patient and imaging data and wrote the manuscript. Pinar Tekturk collected and analyzed patient and imaging data. Fia Cialdella collected and analyzed patient and imaging data. Diane F. van Rappard collected and analyzed patient data. Nicole I. Wolf collected and analyzed patient and imaging data. Cengiz Yalcinkaya collected and analyzed patient and imaging data. Ümran Çetinçelik collected and analyzed patient and imaging data. Ahmad Rajaee collected and analyzed patient and imaging data. Ariana Kariminejad collected and analyzed patient and imaging data. Justyna Paprocka collected and analyzed patient and imaging data. Zuhal Yapici collected and analyzed patient and imaging data. Vlatka Mejaški Bošnjak collected and analyzed patient and imaging data. Marjo S. van der Knaap conceptualized and coordinated the project, collected and analyzed patient and imaging data, and wrote the manuscript.

\section{Acknowledgment}

The authors thank the patients and families for their cooperation and contribution.

\section{Study funding}

This study received financial support from the Optimix Foundation for Scientific Research.

\section{Disclosure}

The authors report no disclosures relevant to the manuscript. Go to Neurology.org/N for full disclosures.

Received September 14, 2017. Accepted in final form January 16, 2018.

\section{References}

1. van der Knaap MS, Barth PG, Stroink H, et al. Leukoencephalopathy with swelling and a discrepantly mild clinical course in eight children. Ann Neurol 1995;37:324-334.
2. Singhal BS, Gursahani RD, Udani VP, Biniwale AA. Megalencephalic leukodystrophy in an Asian Indian ethnic group. Pediatr Neurol 1996;14:291-296.

3. Ridder MC, Boor I, Lodder JC, et al. Megalencephalic leucoencephalopathy with cysts: defect in chloride currents and cell volume regulation. Brain 2011;134: 3342-3354

4. van der Knaap MS, Valk J, Barth PG, Smit LM, van Engelen BG, Tortori Donati P. Leukoencephalopathy with swelling in children and adolescents: MRI patterns and differential diagnosis. Neuroradiology 1995;37:679-686.

5. van der Knaap MS, Lai V, Kohler W, et al. Megalencephalic leukoencephalopathy with cysts without MLC1 defect. Ann Neurol 2010;67:834-837.

6. van der Knaap MS, Boor I, Estevez R. Megalencephalic leukoencephalopathy with subcortical cysts: chronic white matter oedema due to a defect in brain ion and water homoeostasis. Lancet Neurol 2012;11:973-985.

7. Lopez-Hernandez T, Ridder MC, Montolio M, et al. Mutant GlialCAM causes megalencephalic leukoencephalopathy with subcortical cysts, benign familial macrocephaly, and macrocephaly with retardation and autism. Am J Hum Genet 2011;88: $422-432$.

8. Singhal BS, Gorospe JR, Naidu S. Megalencephalic leukoencephalopathy with subcortical cysts. J Child Neurol 2003;18:646-652.

9. Patrono C, Di Giacinto G, Eymard-Pierre E, et al. Genetic heterogeneity of megalencephalic leukoencephalopathy and subcortical cysts. Neurology 2003;61: 534-537.

10. Kariminejad A, Rajaee A, Ashrafi MR, et al. Eight novel mutations in MLC1 from 18 Iranian patients with megalencephalic leukoencephalopathy with subcortical cysts. Eur J Med Genet 2015;58:71-74

11. Cao B, Yan H, Guo M, et al. Ten novel mutations in Chinese patients with megalencephalic leukoencephalopathy with subcortical cysts and a long-term follow-up Research. PLoS One 2016;11:e0157258.

12. Ilja Boor PK, de Groot K, Mejaski-Bosnjak V, et al. Megalencephalic leukoencephalopathy with subcortical cysts: an update and extended mutation analysis of MLC1. Hum Mutat 2006;27:505-512.

13. Schouten JP, McElgunn CJ, Waaijer R, Zwijnenburg D, Diepvens F, Pals G. Relative quantification of 40 nucleic acid sequences by multiplex ligation-dependent probe amplification. Nucleic Acids Res 2002;30:e57.

14. Hidecker MJ, Paneth N, Rosenbaum PL, et al. Developing and validating the communication function classification system for individuals with cerebral palsy. Dev Med Child Neurol 2011;53:704-710.

15. van der Knaap MS, Breiter SN, Naidu S, Hart AA, Valk J. Defining and categorizing leukoencephalopathies of unknown origin: MR imaging approach. Radiology 1999; 213:121-133.

16. Schiffmann R, van der Knaap MS. Invited article: an MRI-based approach to the diagnosis of white matter disorders. Neurology 2009;72:750-759.

17. Dubey M, Bugiani M, Ridder MC, et al. Mice with megalencephalic leukoencephalopathy with cysts: a developmental angle. Ann Neurol 2015;77; 114-131.

18. Lopez-Hernandez T, Sirisi S, Capdevila-Nortes X, et al. Molecular mechanisms of MLC1 and GLIALCAM mutations in megalencephalic leukoencephalopathy with subcortical cysts. Hum Mol Genet 2011;20:3266-3277.

19. Capdevila-Nortes X, Lopez-Hernandez T, Apaja PM, et al. Insights into MLC pathogenesis: GlialCAM is an MLC1 chaperone required for proper activation of volume-regulated anion currents. Hum Mol Genet 2013;22: 4405-4416.

20. Jeworutzki E, Lopez-Hernandez T, Capdevila-Nortes X, et al. GlialCAM, a protein defective in a leukodystrophy, serves as a $\mathrm{ClC}-2 \mathrm{Cl}(-)$ channel auxiliary subunit. Neuron 2012;73:951-961.

21. Wu M, Moh MC, Schwarz H. HepaCAM associates with connexin 43 and enhances its localization in cellular junctions. Sci Rep 2016;6:36218.

22. Depienne C, Bugiani M, Dupuits $\mathrm{C}$, et al. Brain white matter oedema due to $\mathrm{ClC}-2$ chloride channel deficiency: an observational analytical study. Lancet Neurol 2013;12: 659-668.

23. van der Voorn JP, Pouwels PJ, Hart AA, et al. Childhood white matter disorders: quantitative MR imaging and spectroscopy. Radiology 2006;241:510-517.

24. Brimley CJ, Lopez J, van Haren $\mathrm{K}$, et al. National variation in costs and mortality for leukodystrophy patients in US children's hospitals. Pediatr Neurol 2013;49: $156-162$.

25. Dubey M, Brouwers E, Hamilton EM, et al. Seizures and disturbed brain potassium dynamics in the leukodystrophy MLC. Ann Neurol Epub 2018 Feb 21. 


\title{
Megalencephalic leukoencephalopathy with subcortical cysts
}

\author{
Characterization of disease variants
}

\author{
Eline M.C. Hamilton, MD, Pinar Tekturk, MD, Fia Cialdella, BSc, Diane F. van Rappard, MD, Nicole I. Wolf, MD, \\ PhD, Cengiz Yalcinkaya, MD, Ümran Çetinçelik, MD, Ahmad Rajaee, MD, Ariana Kariminejad, MD, \\ Justyna Paprocka, MD, PhD, Zuhal Yapici, MD, Vlatka Mejaški Bošnjak, MD, PhD, \\ and Marjo S. van der Knaap, MD, PhD On behalf of the MLC Research Group
}

Cite as: Neurology ${ }^{\circledR}$ 2018;90:e1395-e1403. doi:10.1212/WNL.0000000000005334

\author{
Correspondence \\ Dr. van der Knaap \\ ms.vanderknaap@vumc.nl
}

\section{Study question}

Are there clinical and MRI characteristics that can distinguish the different subtypes of megalencephalic leukoencephalopathy with subcortical cysts (MLC)?

\section{Summary answer}

MRI findings can distinguish classic MLC from remitting MLC, but distinguishing classic MLC variants resulting from mutations in different genes is not possible.

\section{What is known and what this paper adds}

MLC is a rare disorder with classic forms caused by recessive MLC1 mutations (the MLC1 subtype) or recessive GLIALCAM mutations (the MLC2A subtype) and a remitting form caused by dominant GLIALCAM mutations (the MLC2B subtype). This study provides evidence that MRI findings can distinguish the MLC2B subtype from the MLC2A and MLC1 subtypes.

\section{Participants and setting}

This study analyzed data for 204 patients with classic MLC, including 187 with the MLC1 subtype and 17 with the MLC2A subtype, and 38 patients with the remitting MLC2B subtype. These patients were listed in the Amsterdam Database, which catalogs genetically proven MLC cases from around the world, between January 1991 and January 2017.

\section{Design, size, and duration}

The patients' clinical and MRI data were reviewed by clinicians at the Vrije Universiteit Amsterdam Medical Center. Clinical data were obtained by sending questionnaires to the patients' primary physicians and reviewing medical records.

\section{Primary outcomes}

The primary outcome was the identification of variables that differed between the MLC subtypes.

\section{Main results and the role of chance}

No clinical or MRI variables distinguished the MLC1 subtype from the MLC2A subtype. Relative to the MLC1 and MLC2A
Table Longitudinal changes in cerebral white matter signal abnormalities in cases with adequate MRI data

\begin{tabular}{lll}
\hline Change & Classic MLC & Remitting MLC \\
\hline Normalization & $0 \%(0 / 34)$ & $21 \%(4 / 19)$ \\
\hline Improvement & $6 \%(2 / 34)$ & $74 \%(14 / 19)$ \\
\hline No change & $68 \%(23 / 34)$ & $5 \%(1 / 19)$ \\
\hline Increase & $26 \%(9 / 34)$ & $0 \%(0 / 19)$ \\
\hline
\end{tabular}

Abbreviation: MLC = megalencephalic leukoencephalopathy with subcortical cysts.

subtypes, the MLC2B subtype was associated with a milder clinical presentation, including preservation of motor functions, the absence of delayed-onset cognitive decline, and a lower likelihood of seizures. The MLC2B subtype was also associated with distinctive MRI findings, including mild subarachnoid space enlargement before 2 years of age, a lower likelihood of true cysts, the absence of cerebellar white matter signal abnormalities, and signal normalization and reversal of white matter swelling over time.

\section{Bias, confounding, and other reasons for caution}

Cognitive assessments relied on subjective assessments rather than formal testing. Missing data and inter-observer differences might have introduced bias.

\section{Generalizability to other populations}

Given the rarity of the disease, this study had a large and diverse sample, which favors the generalizability of the results.

\section{Study funding/potential competing interests}

This study was funded by the Optimix Foundation for Scientific Research. The authors report no competing interests. Go to Neurology.org/N for full disclosures. 


\section{Neurology}

\section{Megalencephalic leukoencephalopathy with subcortical cysts: Characterization of disease variants \\ Eline M.C. Hamilton, Pinar Tekturk, Fia Cialdella, et al.}

Neurology 2018;90;e1395-e1403 Published Online before print March 21, 2018

DOI 10.1212/WNL.0000000000005334

\section{This information is current as of March 21, 2018}

\section{Updated Information \&} Services

References

Subspecialty Collections

Permissions \& Licensing

Reprints including high resolution figures, can be found at: http://n.neurology.org/content/90/16/e1395.full

This article cites 24 articles, 2 of which you can access for free at: http://n.neurology.org/content/90/16/e1395.full\#ref-list-1

This article, along with others on similar topics, appears in the following collection(s):

All Genetics

http://n.neurology.org/cgi/collection/all_genetics

All Pediatric

http://n.neurology.org/cgi/collection/all_pediatric

Leukodystrophies

http://n.neurology.org/cgi/collection/leukodystrophies

MRI

http://n.neurology.org/cgi/collection/mri

Information about reproducing this article in parts (figures,tables) or in its entirety can be found online at:

http://www.neurology.org/about/about_the_journal\#permissions

Information about ordering reprints can be found online:

http://n.neurology.org/subscribers/advertise

Neurology ${ }^{\circledR}$ is the official journal of the American Academy of Neurology. Published continuously since 1951 , it is now a weekly with 48 issues per year. Copyright Copyright (C) 2018 The Author(s). Published by Wolters Kluwer Health, Inc. on behalf of the American Academy of Neurology.. All rights reserved. Print ISSN: 0028-3878. Online ISSN: 1526-632X.

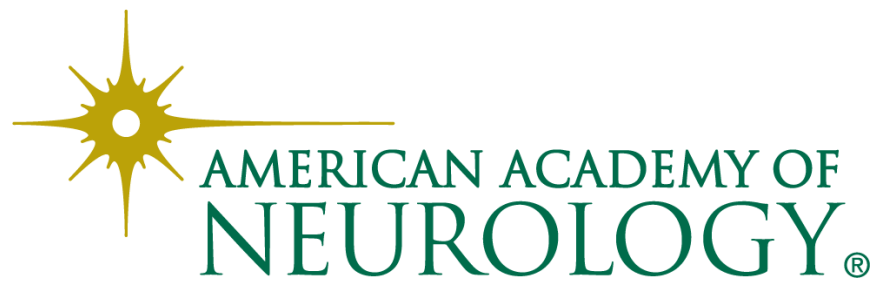

